\title{
AN INVERSE BOUNDARY VALUE PROBLEM FOR SCHRÖDINGER OPERATORS WITH VECTOR POTENTIALS
}

\author{
ZIQI SUN
}

\begin{abstract}
We consider the Schrödinger operator for a magnetic potential $\vec{A}$ and an electric potential $q$, which are supported in a bounded domain in $\mathbb{R}^{n}$ with $n \geq 3$. We prove that knowledge of the Dirichlet to Neumann map associated to the Schrödinger operator determines the magnetic field $\operatorname{rot}(\vec{A})$ and the electric potential $q$ simultaneously, provided $\operatorname{rot}(\vec{A})$ is small in the $L^{\infty}$ topology.
\end{abstract}

\section{INTRODUCTION}

In this paper we consider the Schrödinger operator

$$
H_{\vec{A}, q}=\sum_{j=1}^{n}\left(-i \frac{\partial}{\partial x_{j}}+A_{j}(x)\right)^{2}+q(x),
$$

where $x=\left(x_{1}, x_{2}, \ldots, x_{n}\right) \in \mathbb{R}^{n}, n \geq 2, i=\sqrt{-1}$. The vector function $\vec{A}=\left(A_{1}, A_{2}, \ldots, A_{n}\right)$ is the magnetic potential and the scalar function $q$ is the electric potential. We assume that $A_{j} \in W^{1, \infty}\left(\mathbb{R}^{n}\right), 1 \leq j \leq n, q \in L^{\infty}\left(\mathbb{R}^{n}\right)$, and that they are real-valued.

Let $\Omega$ be a bounded domain in $\mathbb{R}^{n}$ with smooth boundary. If zero is not a Dirichlet eigenvalue of $(1.1)$ on $\Omega$, then for any boundary value $f \in H^{1 / 2}(\partial \Omega)$ there exists a unique solution $u \in H^{1}(\Omega)$ which solves

$$
H_{\vec{A}, q} u=0 \quad \text { in } \Omega \quad \text { and }\left.\quad u\right|_{\partial \Omega}=f .
$$

Variational principles show that the solution $u$ in (1.2) can be obtained by minimizing the functional

$$
\mathbf{I}_{\vec{A}, q}(w)=\int_{\Omega}\left(\nabla w \nabla \bar{w}+\left(\vec{A}^{2}+q\right) w \bar{w}+i \vec{A} \cdot(w \nabla \bar{w}-\bar{w} \nabla w)\right) d x
$$

over functions $w$ with $\left.w\right|_{\partial \Omega}=f$ in $H^{1}(\Omega)$. More precisely,

$$
\mathbf{I}_{\vec{A}, q}(u)=\inf _{\substack{\left.w \in H^{1}(\Omega) \\ w\right|_{i \Omega \Omega}=f}} \mathbf{I}_{\vec{A}, q}(w) .
$$

Received by the editors May 29, 1991.

1991 Mathematics Subject Classification. Primary 35R30; Secondary 35Q40.

Partly supported by NSF DMS 9015621 . 
In terms of boundary value $f$, the functional $\mathbf{I}_{\vec{A}, q}$ can be expressed also as

$$
\mathbf{I}_{\vec{A}, q}(u)=\int_{\partial \Omega} \bar{f} \Lambda_{\vec{A}, q}(f) d s,
$$

where the operator $\Lambda_{\vec{A}, q}$, mapping $H^{1 / 2}(\partial \Omega)$ into $H^{-1 / 2}(\partial \Omega)$, is defined as

$$
\Lambda_{\vec{A}, q}:\left.f \rightarrow \frac{\partial u}{\partial N}\right|_{\partial \Omega}+i(\vec{A} \cdot N) f, \quad f \in H^{1 / 2}(\partial \Omega),
$$

with $u$ solution of (1.2) and $N$ the outer normal on $\partial \Omega$.

The operator $\Lambda_{\vec{A}, q}$, which is the main subject of this paper, is called the Dirichlet to Neumann map of $H_{\vec{A}, q}$ on $\partial \Omega$. In this paper we assume that $\operatorname{supp} \vec{A}, \operatorname{supp} q \subset \bar{\Omega}$. Thus in this case

$$
\Lambda_{\vec{A}, q}(f)=\left.\frac{\partial u}{\partial N}\right|_{\partial \Omega} .
$$

When $\Omega$ is given, the Dirichlet to Neumann map $\Lambda_{\vec{A}, q}$ is uniquely determined by $H_{\vec{A}, q}$, i.e., by potentials $\vec{A}$ and $q$. The problem under discussion in this paper is whether the converse is true. More specifically, we ask whether the potentials $\vec{A}$ and $q$ are uniquely determined by $\Lambda_{\vec{A}, q}$. A resolution to this problem would have important applications to the problem of the inverse scattering at fixed energy. On the other hand, inverse boundary value problems for general elliptic operators are of independent interest. Our study is partly devoted to understand exactly what the Dirichlet to Neumann map does determine if an elliptic operator involves a first-order term. One shall note that a selfadjoint elliptic operator of second order with $\Delta$ as its principal symbol can always be written as a Schrödinger operator with vector potentials.

In recent years significant progress has been made on this problem in the case of $\vec{A}=0$. It has been shown that in dimension $n \geq 3$, an $L^{\infty}$ potential $q$ is uniquely determined by the Dirichlet to Neumann map $\Lambda_{q}$ [NSU]. The $L^{\infty}$ hypothesis on $q$ can even be relaxed to $L^{s}$ with $s>n / 2$ [Ch] and to $L^{n / 2}$ [LN], and the smoothness assumption on $\partial \Omega$ can be relaxed to $C^{1,1}$ [N]. It has also been shown that in dimension $n=2$, a $W^{1, \infty}$ potential $q$ is uniquely determined by $\Lambda_{q}$ provided $q$ is close to zero [SU-II] and close to "most potentials" [SuU-I]. More recently, it has been shown that singularities of an arbitrary two-dimensional potential $q$ are uniquely determined by $\Lambda_{q}$ [SuU-II]. We refer readers to [C, KV-I, KV-II, SU-I, A, I], and [Su-I] for results on the inverse isotropic conductivity problem which is closely related to the problem discussed here and to [NH, N, R], and [W] for applications to inverse scattering.

In the case of $\vec{A} \neq 0$, however, there is an obstruction to uniqueness. In fact a change of the magnetic potential $\vec{A}$ to its gauge equivalence $\overrightarrow{A^{\prime}}=\vec{A}+\nabla g$ for some $g \in W^{1, \infty}$ with $g=\partial g / \partial N=0$ on $\partial \Omega$ would not change the Dirichlet to Neumann map $\Lambda_{\vec{A}, q}$. Indeed, it is a straightforward computation to show that replacing $\vec{A}$ by $\vec{A}^{\prime}$ in (1.1) is equivalent to replacing the solution $u$ in (1.2) by $u^{\prime}=u e^{-i g}$. Since $u^{\prime}$ carries the same boundary value and the normal derivative as $u$, it follows that $\Lambda_{\overrightarrow{A^{\prime}, q}}=\Lambda_{\vec{A}, q}$. 
It is easy to see that the above gauge transformation $\vec{A} \rightarrow \overrightarrow{A^{\prime}}$ preserves the rotation $\operatorname{rot}(\vec{A})=\operatorname{rot}\left(\overrightarrow{A^{\prime}}\right)$, where

$$
\operatorname{rot}(\vec{A})=\sum_{j, l=1}^{n}\left(\frac{\partial A_{l}}{\partial x_{j}}-\frac{\partial A_{j}}{\partial x_{l}}\right) d x_{j} \wedge d x_{l} .
$$

Physically, $\operatorname{rot}(\vec{A})$ is called the magnetic field induced by $\vec{A}$. On the other hand, one shows easily that if $\operatorname{rot}(\vec{A})=\operatorname{rot}\left(\vec{A}^{\prime}\right)$ holds for two magnetic potentials $\vec{A}$ and $\overrightarrow{A^{\prime}}$ satisfying our basic hypotheses and if $\Omega$ is simply connected, then $\vec{A}$ and $\overrightarrow{A^{\prime}}$ are gauge equivalent and therefore $\Lambda_{\vec{A}^{\prime}, q}=\Lambda_{\vec{A}, q}$.

The above analysis shows that in general the best one can expect in the case of $\vec{A} \neq 0$ is that $\Lambda_{\vec{A}, q}$ determines $\operatorname{rot}(\vec{A})$ and $q$ uniquely. The goal of this paper is to show that in dimension $n \geq 3$, and under the a priori hypothesis that $\operatorname{rot}(\vec{A})$ is small in the $L^{\infty}$ topology, $\Lambda_{\vec{A}, q}$ determines $\operatorname{rot}(\vec{A})$ and $q$ uniquely. In what follows we use $W_{\Omega}^{m, \infty}$ to denote the space of functions $f$ in $W^{m, \infty}\left(\mathbb{R}^{n}\right)$ with $\operatorname{supp} f \subset \bar{\Omega}$.

Theorem. Let $\vec{A}_{j} \in W_{\Omega}^{2, \infty}, q_{j} \in L^{\infty}(\Omega), j=1,2$. Assume that zero is not a Dirichlet eigenvalue of $H_{\vec{A}_{j}, q_{j}}, j=1,2$. Then there exists a positive constant $\varepsilon=\varepsilon(\Omega)$ such that if

$$
\left\|\operatorname{rot}\left(\vec{A}_{j}\right)\right\|_{L^{\infty}(\Omega)}<\varepsilon, \quad j=1,2,
$$

and

$$
\Lambda_{\vec{A}_{1}, q_{1}}=\Lambda_{\overrightarrow{A_{2}}, q_{2}},
$$

then

$$
\operatorname{rot}\left(\overrightarrow{A_{1}}\right)=\operatorname{rot}\left(\overrightarrow{A_{2}}\right) \quad \text { and } \quad q_{1}=q_{2} .
$$

In $\S 2$ we shall construct a special class of expotentially growing solutions in the null space of (1.1), which are analogous to the special solutions constructed by Sylvester and Uhlmann in [SU-I]. These solutions shall serve as a basic tool in this paper. The presence of the magnetic potential $\vec{A}$ in (1.1) makes the construction much more difficult especially when $\operatorname{rot}(\vec{A})$ is not small. This is the only reason which leads to the smallness assumption on $\operatorname{rot}(\vec{A})$ in the theorem. If one converts the differential equation $H_{\vec{A}, q} u=0$ into an integral equation using Faddeev's Green's function, one sees that the set of exceptional points for that integral equation may not be bounded in $\mathbb{C}^{n}$ when $\operatorname{rot}(\vec{A})$ is not small $[\mathrm{NH}]$. Therefore it remains as an open question whether such solutions can still be constructed when $\operatorname{rot}(\vec{A})$ is large.

Section 3 is devoted to establish an orthogonality identity which relates $\vec{A}$ and $q$ with $\Lambda_{\vec{A}, q}$.

Section 4 is the heart of the proof. The main difficulty, which one did not encounter in the case of $\vec{A}=0$ treated in [NSU], is that one has to determine $\operatorname{rot}(\vec{A})$ from a nonlinear integral functional rather than the Fourier transform of $\operatorname{rot}(\vec{A})$. Once $\operatorname{rot}(\vec{A})$ has been recovered from $\Lambda_{\vec{A}, q}$, we can go further to recover $q$ using gauge invariant property of $\Lambda_{\vec{A}, q}$ and the method in [NSU]. 
We remark that this method does not apply in dimension 2 . We shall study the two-dimensional case in a forthcoming paper [Su-II].

\section{CONSTRUCTION OF SOlUtions}

Following the idea of the geometric optics construction of solutions to hyperbolic equations, we look for solutions of the form given below in the null space of $H_{\vec{A}, q}$.

$$
u(x, \xi)=e^{\xi x+\phi(x, \xi)}(1+\omega(x, \xi)),
$$

where $\xi \in \mathbb{C}^{n}$ is a complex vector satisfying $\xi \cdot \xi=0$ and the function $\omega(x, \xi)$ behaves like $|\xi|^{-1}$ as $|\xi|$ tends to $\infty$ in an appropriate function space. We shall show that it is always possible to construct such solutions provided $\|\operatorname{rot}(\vec{A})\|_{L^{\infty}(\Omega)}$ is sufficiently small and $|\xi|$ is sufficiently large.

Substituting (2.1) into the equation $H_{\vec{A}, q} u=0$ and equating coefficients of powers of $|\xi|$ to zero, we get two equations

$$
\begin{gathered}
\xi \cdot \nabla \phi=-i \xi \cdot \vec{A}, \\
\Delta \omega+2(\xi+\nabla \phi+i \vec{A}) \cdot \nabla \omega-G \omega=G,
\end{gathered}
$$

where

$$
G=\vec{A}^{2}-i \nabla \cdot \vec{A}+q-2 i \vec{A} \cdot \nabla \phi-\nabla \phi \cdot \nabla \phi-\Delta \phi .
$$

We divide the rest of the section into two parts, where $\phi$ and $\omega$ will be constructed separately. In what follows we assume

$$
|\xi| \geq 1, \quad \vec{A} \in W_{\Omega}^{2, \infty}, \quad q \in L^{\infty}\left(\mathbb{R}^{n}\right), \quad \operatorname{supp} q \subset \bar{\Omega} .
$$

2.1. Construction of $\phi$. Fourier transforming (2.2) gives

$$
-i \xi \cdot \eta \hat{\phi}(\eta, \xi /|\xi|)=-i \xi \cdot \vec{A}^{\wedge}(\eta),
$$

where $\eta=\left(\eta_{1}, \eta_{2}, \ldots, \eta_{n}\right)$ is the dual coordinates and $\wedge$ denotes the Fourier transform with respect to $x$. We denote by $\vee$ its inverse. We construct

$$
\phi\left(x, \frac{\xi}{|\xi|}\right)=\left(\frac{\xi \cdot \overrightarrow{A^{\wedge}}(\eta)}{\xi \cdot \eta}\right)^{\vee}=(2 \pi)^{-n} \int_{\mathbb{R}^{n}} e^{-i x \eta}\left(\frac{\xi \cdot \overrightarrow{A^{\wedge}}(\eta)}{\xi \cdot \eta}\right) d \eta .
$$

We shall show that the solution $\phi(x, \xi)$ has the following three properties:

$$
\begin{gathered}
\|\phi(\cdot, \xi /|\xi|)\|_{W^{2, \infty}(\Omega)} \leq C\|\vec{A}\|_{W_{\Omega}^{2, \infty}}, \\
\|\nabla \phi+i \vec{A}\|_{L^{\infty}(\Omega)} \leq C\|\operatorname{rot}(\vec{A})\|_{L^{\infty}(\Omega)},
\end{gathered}
$$

If $\xi(s):(a, b) \rightarrow \mathbb{C}^{n}$ is a differentiable map with $\xi(s) \cdot \xi(s)=0$ and $|\xi(s)| \geq 1$ for all $s$, then $s \rightarrow \phi(\cdot, \xi(s) /|\xi(s)|)$ is differentiable as a map from $(a, b)$ to $L^{\infty}(\Omega)$.

The constant $C$ involved in (2.7)-(2.9) depends only on $\Omega$. 
Lemma 2.1. Let $\Omega$ be a bounded domain in $\mathbb{R}^{n}, n \geq 2$. Then

$$
L(f)=(2 \pi)^{-n} \int_{\mathbb{R}^{n}} e^{-i x \eta}\left(\frac{\hat{f}(\eta)}{\eta_{1}+i \eta_{2}}\right) d \eta
$$

defines a bounded map from $W_{\Omega}^{m, \infty}$ to $W^{m, \infty}(\Omega)$ for any nonnegative integer $m$.

Proof. Rewriting (2.10) in terms of convolution respect to variable $\left(x_{1}, x_{2}\right)$, we get

$$
L(f)=\int_{\mathbb{R}^{2}} \frac{f\left(x_{1}-z_{1}, x_{2}-z_{2}, x_{3}, \ldots, x_{n}\right)}{z_{1}+i z_{2}} d z_{1} d z_{2}
$$

and thus

$$
\|L(f)\|_{L^{\infty}(\Omega \cap T)} \leq C\|f\|_{L^{\infty}(\Omega \cap T)}
$$

for any two-dimensional plane $T$ that is parallel to $\left(x_{1}, x_{2}\right)$-plane. Therefore

$$
\|L(f)\|_{L^{\infty}(\Omega)} \leq C\|f\|_{L^{\infty}(\Omega)} .
$$

This proves Lemma 2.1 in the case of $m=0$. Differentiating both sides of (2.11) and repeating using (2.13) yield desired results.

Proof of (2.7). Without loss of generality, we assume that $\xi=\gamma_{1}+i \gamma_{2}$, where $\gamma_{1}, \gamma_{2} \in \mathbb{R}^{n},\left|\gamma_{1}\right|=\left|\gamma_{2}\right|=1$, and $\gamma_{1} \cdot \gamma_{2}=0$. Making a rotation of coordinates if necessary one can rewrite (2.6) in terms of convolution as follows:

$$
\phi\left(x, \frac{\xi}{|\xi|}\right)=\int_{\mathbb{R}^{2}} \frac{\xi \cdot \vec{A}\left(x-\left(z_{1} \gamma_{1}+z_{2} \gamma_{2}\right)\right)}{z_{1}+i z_{2}} d z_{1} d z_{2} .
$$

Clearly, (2.7) follows from Lemma 2.1. If $\xi(s)=\gamma_{1}(s)+i \gamma_{2}(s)$ is differentiable in $s$ and $\left|\gamma_{1}(s)\right|=\left|\gamma_{2}(s)\right|=1$ for all $s \in(a, b)$, then for a fixed $x \in \Omega$,

$$
\begin{aligned}
\dot{\phi}\left(x, \frac{\xi}{|\xi|}\right)= & \int_{\mathbb{R}^{2}} \frac{\dot{\xi} \cdot \vec{A}\left(x-\left(z_{1} \gamma_{1}+z_{2} \gamma_{2}\right)\right)}{z_{1}+i z_{2}} d z_{1} d z_{2} \\
& +\int_{\mathbb{R}^{2}} \frac{\left(z_{1} \dot{\gamma}_{1}+z_{2} \dot{\gamma}_{2}\right) \cdot \nabla(\xi \cdot \vec{A})\left(x-\left(z_{1} \gamma_{1}+z_{2} \gamma_{2}\right)\right)}{z_{1}+i z_{2}} d z_{1} d z_{2},
\end{aligned}
$$

where the dot means $d / d s$. Using Lemma 2.1 again and noting that $\operatorname{supp} \vec{A} \subset$ $\bar{\Omega}$, one sees that the right-hand side of $(2.15)$ is a function in $L^{\infty}(\Omega)$. Hence (2.8) follows.

By a change of coordinates we need only to prove (2.9) in the case that $\gamma_{1}=(1,0, \ldots, 0)$ and $\gamma_{2}=(0,1, \ldots, 0)$. In this case

$$
\nabla \phi+i \vec{A}=\left(-i \eta \frac{\widehat{A_{1}}+i \widehat{A_{2}}}{\eta_{1}+i \eta_{2}}+i \overrightarrow{A^{\wedge}}\right)^{\vee} .
$$

We now compute explicitly the components of $\nabla \phi+i \vec{A}$. We have

$$
\begin{aligned}
\left(\frac{-\eta_{1}\left(\widehat{A_{1}}+i \widehat{A_{2}}\right)}{\eta_{1}+i \eta_{2}}+i \widehat{A_{1}}\right)^{\vee} & =\left(\frac{-i \eta_{1}\left(\widehat{A_{1}}+i \widehat{A_{2}}\right)+i \widehat{A_{1}}\left(\eta_{1}+i \eta_{2}\right)}{\eta_{1}+i \eta_{2}}\right)^{\vee} \\
& =i L\left(\frac{\partial A_{2}}{\partial x_{1}}-\frac{\partial A_{1}}{\partial x_{2}}\right)
\end{aligned}
$$




$$
\begin{aligned}
\left(\frac{-i \eta_{2}\left(\widehat{A_{1}}+i \widehat{A_{2}}\right)}{\eta_{1}+i \eta_{2}}+i \widehat{A_{2}}\right)^{\vee} & =\left(\frac{-i \eta_{2}\left(\widehat{A_{1}}+i \widehat{A_{2}}\right)+i \widehat{A_{2}}\left(\eta_{1}+i \eta_{2}\right)}{\eta_{1}+i \eta_{2}}\right)^{\vee} \\
& =L\left(\frac{\partial A_{1}}{\partial x_{2}}-\frac{\partial A_{2}}{\partial x_{1}}\right)
\end{aligned}
$$

and for $3 \leq j \leq n$,

$$
\begin{aligned}
\left(\frac{-i \eta_{j}\left(\widehat{A_{1}}+i \widehat{A_{2}}\right)}{\eta_{1}+i \eta_{2}}+i \widehat{A_{j}}\right)^{\vee} & =\left(\frac{-i \eta_{j}\left(\widehat{A_{1}}+i \widehat{A_{2}}\right)+i \widehat{A_{j}}\left(\eta_{1}+i \eta_{2}\right)}{\eta_{1}+i \eta_{2}}\right)^{\vee} \\
& =L\left(\frac{\partial A_{1}}{\partial x_{j}}-\frac{\partial A_{j}}{\partial x_{1}}\right)-i L\left(\frac{\partial A_{2}}{\partial x_{j}}-\frac{\partial A_{j}}{\partial x_{2}}\right) .
\end{aligned}
$$

Applying Lemma 2.1 to $(2.16)-(2.18)$ we get (2.8).

\subsection{Construction of $\omega$.}

Proposition 2.2. Let $\vec{A}$ and $q$ be potential functions satisfying (2.5). Then there exist positive constants $\delta=\delta(\Omega)$ and $K$ such that if $\left\|\operatorname{rot}\left(\vec{A}_{j}\right)\right\|_{L^{\infty}(\Omega)}<\varepsilon$ and $|\xi|>K$, then equation (2.3) has a solution $\omega \in H^{1}(\Omega)$. Moreover,

$$
\|\omega\|_{L^{2}(\Omega)} \leq C|\xi|^{-1}
$$

and

$$
\|\nabla \omega\|_{L^{2}(\Omega)} \leq C,
$$

where $K$ and $C$ depend only on $\Omega,\|\vec{A}\|_{W^{2, \infty}(\Omega)}$, and $\|q\|_{L^{\infty}(\Omega)}$.

The proof of this proposition is based on the following two lemmas.

Lemma 2.3. Let $L_{\xi}=\Delta+2 \xi \cdot \nabla$. Then the operator $L_{\xi}$ admits a bounded inverse $L_{\xi}^{-1}: L^{2}(\Omega) \rightarrow H^{1}(\Omega)$. If $f \in L^{\infty}(\Omega)$ and $v=L_{\xi}^{-1}(f) \in H^{1}(\Omega)$, then

$$
\begin{gathered}
\|v\|_{L^{2}(\Omega)} \leq C|\xi|^{-1}\|f\|_{L^{2}(\Omega)}, \\
\|\nabla v\|_{L^{2}(\Omega)} \leq C\|f\|_{L^{2}(\Omega)},
\end{gathered}
$$

where $C$ depends only on $\Omega$.

Proof. The existence of $L_{\xi}^{-1}$ as well as the estimate (2.21) follows from a fundamental result obtained by Sylvester and Uhlmann [SU-I]. (Also see [I] for a more direct proof.) The estimate (2.22) was proven in the two dimensional case [SU-II]. We now give a proof of $(2.22)$ in the case of $n \geq 3$.

Extending $f$ to be zero outside $\Omega$ and letting $\Omega^{\prime}$ be a bounded domain that contains $\Omega$ we can construct a solution $v$ satisfying (2.21). We shall show that the following estimate must hold:

$$
\|\nabla v\|_{L^{2}(\Omega)}^{2} \leq C\left(|\xi|^{2}\|v\|_{L^{2}\left(\Omega^{\prime}\right)}^{2}+\|f\|_{L^{2}(\Omega)}^{2},\right.
$$

where $C=\left(\Omega, \Omega^{\prime}\right)$. Clearly, the restriction of $v$ to $\Omega$ is a solution satisfying (2.21) and (2.22).

Let $\chi \in C_{0}^{\infty}\left(\Omega^{\prime}\right)$ so that $\chi(x)=1$ for $x \in \Omega$ and $0 \leq \chi(x) \leq 1$ for $x \in \Omega^{\prime} \backslash \Omega$. Let $u=\chi v$. It follows from an elliptic regularity theorem that $u$ is a $H^{2}(\Omega)$ solution of the equation

$$
\Delta u+2 \xi \cdot \nabla u=f \chi+v \Delta \chi+2 \nabla v \nabla \chi+2 v \xi \cdot \nabla \chi .
$$


Multiplying $\bar{u}$ to both sides of (2.24) and integrating by parts we get

$$
\begin{aligned}
\int_{\Omega^{\prime}}|\nabla u|^{2} d x \leq & 2 \int_{\Omega^{\prime}}(|\bar{u} \xi \cdot \nabla u|+|f \bar{u} \chi+\bar{u} \chi+\bar{u} v \Delta \chi+2 \bar{u} v \xi \cdot \nabla \chi|) d x \\
& +2\left|\int_{\Omega^{\prime}} \bar{u} \nabla v \nabla \chi d x\right| \\
= & I_{1}+I_{2} .
\end{aligned}
$$

Denoting $M=\|\chi\|_{C^{2}\left(\Omega^{\prime}\right)}$ and using Schwartz's inequality we get

$$
\begin{aligned}
I_{1} \leq & \left(4|\xi|^{2}+M\left(1+|\xi|^{2}\right)\right) \int_{\Omega^{\prime}}|u|^{2} d x \\
& +\frac{1}{4} \int_{\Omega^{\prime}}|\nabla u|^{2} d x+M\left(2 \int_{\Omega^{\prime}}|v|^{2} d x+\int_{\Omega^{\prime}}|f|^{2} d x\right) .
\end{aligned}
$$

Using integration by parts one has

$$
\int_{\Omega^{\prime}} \bar{u} \nabla v \nabla u d x=-\int_{\Omega^{\prime}} v(\bar{u} \Delta \chi+\nabla \chi \nabla u) d x .
$$

Hence

$$
\begin{aligned}
I_{2} & \leq 2 M \int_{\Omega^{\prime}}(|v \bar{u}|+|v \nabla \bar{u}|) d x \\
& \leq\left(M+4 M^{2}\right) \int_{\Omega^{\prime}}|v|^{2} d x+M \int_{\Omega^{\prime}}|u|^{2} d x+\frac{1}{4} \int_{\Omega^{\prime}}|\nabla u|^{2} d x .
\end{aligned}
$$

Combining (2.25) with (2.26) and (2.27) yields

$$
\begin{aligned}
\frac{1}{2}\|\nabla v\|_{L^{2}\left(\Omega^{\prime}\right)}^{2} \leq & \left(|\xi|^{2}(M+4)+2 M\right) \int_{\Omega^{\prime}}|u|^{2} d x \\
& +\left(3 M+4 M^{2}\right) \int_{\Omega^{\prime}}|v|^{2} d x+M \int_{\Omega^{\prime}}|f|^{2} d x .
\end{aligned}
$$

Since $u=v$ in $\Omega$ and $|u| \leq|v|$ in $\Omega^{\prime}$, it follows from (2.28) that

$$
\|\nabla v\|_{L^{2}(\Omega)}^{2} \leq 2\left(|\xi|(M+4)+5 M+4 M^{2}\right)\|v\|_{L^{2}\left(\Omega^{\prime}\right)}^{2}+M\|f\|_{L^{2}\left(\Omega^{\prime}\right)}^{2} .
$$

This leads to (2.23) immediately.

We set $\widetilde{G}=G_{\chi_{\Omega}}$ and $\tilde{\phi}=\phi \chi_{\Omega}$, with $G$ and $\phi$ as in (2.2) and (2.4), where $\chi_{\Omega}$ is the indicator function of $\Omega$. To solve (2.3) it suffices to solve

$$
\left(L_{\xi, \vec{A}}-\widetilde{G}\right) \omega=\widetilde{G}
$$

where

$$
L_{\xi, \vec{A}}=L_{\xi}+(\nabla \tilde{\phi}+i \vec{A}) \cdot \nabla=\Delta+2(\xi+\nabla \tilde{\phi}+i \vec{A}) \cdot \nabla
$$

Lemma 2.4. If $\|\operatorname{rot} \vec{A}\|_{L^{\infty}(\Omega)}$ is sufficiently small, then $L_{\xi, \vec{A}}$ has a bounded inverse $L_{\xi, \vec{A}}^{-1}: L^{2}(\Omega) \rightarrow H^{1}(\Omega)$. Moreover, if $f \in L^{2}(\Omega)$ and $v=L_{\xi, \vec{A}}^{-1}(f)$, then

$$
\begin{gathered}
\|v\|_{L^{2}(\Omega)} \leq C|\xi|^{-1}\|f\|_{L^{2}(\Omega)}, \\
\|\nabla v\|_{L^{2}(\Omega)} \leq C\|f\|_{L^{2}(\Omega)},
\end{gathered}
$$

where $C$ depends only on $\Omega$ and $\|\operatorname{rot} \vec{A}\|_{L^{x}(\Omega)}$. 
Proof. Applying $L_{\xi}^{-1}$ to both sides of $L_{\xi, \vec{A}} v=f$ yields an integral equation

$$
\left(I+2 F_{1}\right) v=L_{\xi}^{-1} f,
$$

where

$$
F_{1}=L_{\xi}^{-1} \circ(\nabla \tilde{\phi}+i \vec{A}) \circ \nabla,
$$

where $I$ denotes the identity operator and ( ) denotes the multiplication operator.

From Lemma 2.3 one sees clearly that the right-hand side of $(2.32)$ is in $H^{1}(\Omega)$. Moreover,

$$
\begin{gathered}
\left\|L_{\xi}^{-1} f\right\|_{L^{2}(\Omega)} \leq C|\xi|^{-1}\|f\|_{L^{2}(\Omega)}, \\
\left\|L_{\xi}^{-1} f\right\|_{H^{1}(\Omega)} \leq C\|f\|_{L^{2}(\Omega)} .
\end{gathered}
$$

From (2.8), (2.21), and (2.22) we have that $F_{1}$ maps $H^{1}(\Omega)$ into $H^{1}(\Omega)$ and moreover,

$$
\left\|F_{1}\right\|_{H^{1}, H^{1}} \leq C\|\operatorname{rot} \vec{A}\|_{L^{\infty}(\Omega)}
$$

where $C$ depends only on $\Omega$. Thus, if we view (2.32) as an integral equation in $H^{1}(\Omega)$ and use (2.34) and (2.35), we conclude that (2.32) has a unique solution $v \in H^{1}(\Omega)$ provided $\|\operatorname{rot} \vec{A}\|_{L^{\infty}(\Omega)}$ is sufficiently small and thus (2.31) follows. Clearly,

$$
v=L_{\xi}^{-1}(f+(\nabla \tilde{\phi}+i \vec{A}) \circ \nabla v) .
$$

Hence by (2.21),

$$
\|v\|_{L^{2}(\Omega)} \leq C|\xi|^{-1}\left(\|f\|_{L^{2}(\Omega)}+\|\operatorname{rot} \vec{A}\|_{L^{\infty}(\Omega)}\|\nabla v\|_{L^{2}(\Omega)}\right) .
$$

This estimate together with (2.31) leads to (2.30).

Proof of Proposition 2.2. Applying $L_{\xi, \vec{A}}^{-1}$ to both sides of (2.29) yields an integral equation for $\omega$ :

$$
\left(I+F_{2}\right) \omega=L_{\xi, \tilde{A}^{-1}}^{-1}(\widetilde{G})
$$

where

$$
F_{2}=L_{\xi, \vec{A}^{-1}}^{-1}(\widetilde{G})
$$

By Lemma 2.4 one sees clearly that the right-hand side of $(2.36)$ is in $H^{1}(\Omega)$. Moreover,

$$
\begin{gathered}
\left\|L_{\xi, \vec{A}}^{-1} \widetilde{G}\right\|_{L^{2}(\Omega)} \leq C|\xi|^{-1}\|\widetilde{G}\|_{L^{2}(\Omega)}, \\
\left\|L_{\xi, \vec{A}}^{-1} \widetilde{G}\right\|_{H^{1}(\Omega)} \leq C\|\widetilde{G}\|_{L^{2}(\Omega)} .
\end{gathered}
$$

We view (2.36) as an integral equation in $L^{2}(\Omega)$. From (2.30) and (2.31) we see clearly that $F_{2}$ maps $L^{2}(\Omega)$ into $L^{2}(\Omega)$ and moreover,

$$
\left\|F_{2}\right\|_{L^{2}, L^{2}} \leq C\|\widetilde{G}\|_{L^{\infty}(\Omega)}|\xi|^{-1},
$$


where $C$ depends only on $\Omega$ and $\|\operatorname{rot} \vec{A}\|_{L^{\infty}(\Omega)}$. Therefore (2.36) has a unique $L^{2}$ solution $\omega$ provided $|\xi|$ is large enough. Using (2.37) one sees that $\omega$ satisfies (2.19). On the other hand (2.36) can be rewritten as

$$
\omega=L_{\xi, \widetilde{A}}^{-1}(\widetilde{G}(1+\omega)) \text {. }
$$

Now it follows from (2.31) and (2.19) that the $L^{2}$ solution $\omega$ is also a $H^{1}$ solution of (2.36). Moreover,

from which (2.20) follows.

$$
\|\omega\|_{H^{1}(\Omega)} \leq C\|G\|_{L^{\infty}(\Omega)}\left(1+\|\omega\|_{L^{2}(\Omega)}\right)
$$

\section{AN IDENTITY}

The main purpose of this section is to present an orthogonality identity which relates potential functions $\vec{A}$ and $q$ to the Dirichlet to Neumann map $\Lambda_{\vec{A}, q}$. Proposition 3.1. Let $\overrightarrow{A_{j}}$ and $q_{j}, j=1,2$, be potential functions satisfying (2.5). Then

$$
\begin{aligned}
& i \int_{\Omega}\left(\overrightarrow{A_{1}}-\vec{A}_{2}\right) \cdot\left(u_{1} \nabla \bar{u}_{2}-\bar{u}_{2} \nabla u_{1}\right) d x+\int_{\Omega}\left(\vec{A}_{1}^{2}-\vec{A}_{2}^{2}+q_{1}-q_{2}\right) u_{1} \bar{u}_{2} d x \\
& \quad=-\int_{\partial \Omega} \bar{u}_{2}\left(\Lambda_{\vec{A}_{1}, q_{1}}-\Lambda_{\vec{A}_{2}, q_{2}}\right) u_{1} d s,
\end{aligned}
$$

holds for arbitrary $u_{j}$ solution of $H_{\vec{A}_{j}, q_{j}} u_{j}=0, j=1,2$.

Proof. We have

$$
\begin{array}{ll}
\Delta u_{1}+2 i \vec{A}_{1} \cdot \nabla u_{1}-Q_{1} u_{1}=0 & \text { in } \Omega, \\
\Delta \bar{u}_{2}-2 i \vec{A}_{2} \cdot \nabla \bar{u}_{2}-\bar{Q}_{2} \bar{u}_{2}=0 & \text { in } \Omega,
\end{array}
$$

where

$$
Q_{j}=\vec{A}_{j}^{2}-i \nabla \cdot \vec{A}_{j}+q_{j}, \quad j=1,2 .
$$

Multiplying (3.1) by $\bar{u}_{2}$ and (3.2) by $u_{1}$ and then adding them, we get

$$
\int_{\Omega}\left(\bar{u}_{2} \Delta u_{1}+u_{1} \Delta \bar{u}_{2}+2 i \bar{u}_{2} \vec{A}_{1} \cdot \nabla u_{1}-2 i u_{1} \vec{A}_{2} \cdot \nabla \bar{u}_{2}-Q_{1} \bar{u}_{2} u_{1}-\bar{Q}_{2} u_{1} \bar{u}_{2}\right) d x=0 .
$$

Integrating by parts yields

$$
\begin{gathered}
-2 i \int_{\Omega} u_{1}\left(\overrightarrow{A_{1}}-\vec{A}_{2}\right) \cdot \nabla \bar{u}_{2} d x-\int_{\Omega} u_{1}\left(Q_{1}-\bar{Q}_{2}\right) \bar{u}_{2} d x-2 i \int_{\Omega} \nabla \cdot \vec{A}_{1} u_{1} \bar{u}_{2} d x \\
+\int_{\partial \Omega}\left(\frac{\partial u_{1}}{\partial N} \bar{u}_{2}-\frac{\partial \bar{u}_{2}}{\partial N} u_{1}+2 i\left(\vec{A}_{1} \cdot N\right) u_{1} \bar{u}_{2}\right) d s=0 .
\end{gathered}
$$

Exchanging positions of $u_{j}$ and $\overrightarrow{A_{j}}, j=1,2$, in (3.4) and then taking the complex conjugate, we get

$$
\begin{gathered}
-2 i \int_{\Omega} \bar{u}_{2}\left(\overrightarrow{A_{1}}-\overrightarrow{A_{2}}\right) \cdot \nabla u_{1} d x+\int_{\Omega} u_{1}\left(Q_{1}-\bar{Q}_{2}\right) \bar{u}_{2} d x+2 i \int_{\Omega} \nabla \cdot \vec{A}_{2} u_{1} \bar{u}_{2} d x \\
+\int_{\partial \Omega}\left(\frac{\partial u_{1}}{\partial N} \bar{u}_{2}+\frac{\partial \bar{u}_{2}}{\partial N} u_{1}+2 i\left(\vec{A}_{2} \cdot N\right) u_{1} \bar{u}_{2}\right) d s=0 .
\end{gathered}
$$


Substracting (3.5) from (3.4) and using (3.3) we get

$$
\begin{gathered}
-i \int_{\Omega}\left(\overrightarrow{A_{1}}-\overrightarrow{A_{2}}\right) \cdot\left(u_{1} \nabla \bar{u}_{2}-\bar{u}_{2} \nabla u_{1}\right) d x-\int_{\Omega}\left(\vec{A}_{1}^{2}-\vec{A}_{2}^{2}+q_{1}-q_{2}\right) u_{1} \bar{u}_{2} d x \\
+\int_{\partial \Omega}\left(\frac{\partial u_{1}}{\partial N} \bar{u}_{2}-\frac{\partial \bar{u}_{2}}{\partial N} u_{1}+2 i\left(\left(\overrightarrow{A_{1}}+\overrightarrow{A_{2}}\right) \cdot N\right) u_{1} \bar{u}_{2}\right) d s=0 .
\end{gathered}
$$

Since (3.6) holds for any $\vec{A}_{j}$ and $q_{j}, j=1,2$, it holds in particular for $\overrightarrow{A_{1}}=\overrightarrow{A_{2}}$ and $q_{1}=q_{2}$. In this case $(3.6)$ becomes

$$
\int_{\partial \Omega}\left(\frac{\partial u}{\partial N} \bar{v}-\frac{\partial \bar{v}}{\partial N} u+4 i\left(\overrightarrow{A_{2}} \cdot N\right) u \bar{v}\right) d s=0,
$$

where $u$ and $v$ satisfy

$$
\begin{gathered}
H_{\overrightarrow{A_{2}}, q_{2}} u=H_{\overrightarrow{A_{2}}, q_{2}} v=0 \text { in } \Omega, \\
\left.v\right|_{\partial \Omega}=\left.u_{2}\right|_{\partial \Omega} \text { and }\left.u\right|_{\partial \Omega}=\left.u_{1}\right|_{\partial \Omega} .
\end{gathered}
$$

Hence

$$
\int_{\partial \Omega} \frac{\partial \bar{u}_{2}}{\partial N} u_{1} d x=\int_{\partial \Omega}\left(\frac{\partial u}{\partial N} \bar{u}_{2}+4 i\left(\vec{A}_{2} \cdot N\right) u_{1} \bar{u}_{2}\right) d s .
$$

Using (3.8) and recalling (1.5) we find that the third integral in (3.6) is equal to

$$
\begin{gathered}
\int_{\partial \Omega}\left(\bar{u}_{2}\left(\frac{\partial u_{1}}{\partial N}-\frac{\partial u}{\partial N}\right)+2 i\left(\left(\overrightarrow{A_{1}}-\overrightarrow{A_{2}}\right) \cdot N\right) u_{1} \bar{u}_{2}\right) d s \\
=\int_{\partial \Omega} \bar{u}_{2}\left(\Lambda_{\vec{A}_{1}, q_{1}}-\Lambda_{\vec{A}_{2}, q_{2}}\right) u_{1} d s
\end{gathered}
$$

from which Proposition 3.1 follows.

Corollary 3.2. Let $\overrightarrow{A_{j}}$ and $q_{j}, j=1,2$, be potential functions satisfying (2.5). Assume that $\Lambda_{\vec{A}_{1}, q_{1}}=\Lambda_{\vec{A}_{2}, q_{2}}$. Then

$$
i \int_{\Omega}\left(\overrightarrow{A_{1}}-\overrightarrow{A_{2}}\right) \cdot\left(u_{1} \nabla \bar{u}_{2}-\bar{u}_{2} \nabla u_{1}\right) d x+\int_{\Omega}\left(\vec{A}_{1}^{2}-\vec{A}_{2}^{2}+q_{1}-q_{2}\right) u_{1} \bar{u}_{2} d x=0
$$

holds for arbitrary $u_{j}$ solution of $H_{\vec{A}_{j}, q_{j}} u_{j}=0, j=1,2$.

In the rest of this section we shall replace $u_{j}$ in (3.9) with the exponentially growing solutions constructed in the previous section.

Let $k, \gamma_{1}$, and $\gamma_{2}$ be three mutually orthogonal vectors in $\mathbb{R}^{n}$ with $\left|\gamma_{1}\right|=$ $\left|\gamma_{2}\right|=1$. Let $\zeta, \xi \in \mathbb{C}^{n}$ be given by

$$
\zeta=\gamma_{1}+i \gamma_{2}, \quad \xi=s \zeta+g(s, k) \gamma_{1},
$$

where $s$ is a positive real parameter and

$$
g(s, k)=2^{-1}|k|^{2}\left(\left(|k|^{2}+4 s^{2}\right)^{1 / 2}+4 s\right)^{-1} .
$$

Let $\xi_{1}, \xi_{2} \in \mathbb{C}^{n}$ be given by

$$
\xi_{1}=i k / 2+\xi, \quad \bar{\xi}_{2}=i k / 2-\xi .
$$

One checks directly that

$$
\begin{aligned}
& \xi_{1} \cdot \xi_{1}=\xi_{2} \cdot \xi_{2}=0, \quad \xi_{1}+\bar{\xi}_{2}=i k, \quad \xi_{1}-\bar{\xi}_{2}=2 \xi \\
& \xi_{1} / s \rightarrow \zeta, \quad \bar{\xi}_{2} / s \rightarrow-\zeta, \quad \xi / s \rightarrow \zeta, \quad \text { as } s \rightarrow x
\end{aligned}
$$


Following the construction in $\S 2$ we can construct

$$
u_{j}\left(x, \xi_{j}\right)=e^{\xi_{j} \cdot x+\phi_{j}\left(x, \xi_{j} /\left|\xi_{j}\right|\right)}\left(1+\omega_{j}\left(x, \xi_{j}\right)\right)
$$

solution of $H_{\vec{A}_{j}, q_{j}} u_{j}=0, j=1,2$, where $\phi_{j}$ solves

$$
\xi_{j} \cdot \nabla \phi_{j}=-i \xi_{j} \cdot \vec{A}_{j}, \quad j=1,2,
$$

and $\omega_{j}, j=1,2$, satisfies

$$
\left\|\omega_{j}\right\|_{L^{2}(\Omega)} \leq C\left|\xi_{j}\right|^{-1}
$$

and

$$
\left\|\nabla \omega_{j}\right\|_{L^{2}(\Omega)} \leq C,
$$

where $C$ depends only on $\Omega,\left\|\overrightarrow{A_{j}}\right\|_{W^{2}, \infty(\Omega)}$, and $\left\|q_{j}\right\|_{L^{\infty}(\Omega)}, j=1,2$.

Substituting (3.15) into (3.9) yields

$$
F_{1}+F_{2}=0 \text {, }
$$

where $F_{1}$ and $F_{2}$ are functions of $s, k, \gamma_{1}$, and $\gamma_{2}$ and they are given by the following formulas:

$$
\begin{gathered}
F_{1}=-2 i \int_{\Omega} e^{i k x+\phi_{1}+\bar{\phi}_{2}} \xi \cdot\left(\overrightarrow{A_{1}}-\overrightarrow{A_{2}}\right) d x \\
F_{2}=i \int_{\Omega} e^{i k x+\phi_{1}+\bar{\phi}_{2}}\left(\left(\overrightarrow{A_{1}}-\overrightarrow{A_{2}}\right) \cdot \nabla\left(\bar{\phi}_{2}-\phi_{1}\right)-i\left(\vec{A}_{1}^{2}-\vec{A}_{2}^{2}+q_{1}-q_{2}\right)\right) d x \\
-2 i \int_{\Omega} e^{i k x+\phi_{1}+\bar{\phi}_{2}} \xi \cdot\left(\overrightarrow{A_{1}}-\overrightarrow{A_{2}}\right)\left(\omega_{1}+\bar{\omega}_{2}+\omega_{1} \bar{\omega}_{2}\right) d x \\
+i \int_{\Omega} e^{i k x+\phi_{1}+\bar{\phi}_{2}}\left(\overrightarrow{A_{1}}-\overrightarrow{A_{2}}\right) \cdot\left(\nabla \bar{\omega}_{2}-\nabla \omega_{1}+\omega_{1} \nabla \bar{\omega}_{2}-\bar{\omega}_{2} \nabla \omega_{1}\right) d x \\
+i \int_{\Omega} e^{i k x+\phi_{1}+\bar{\phi}_{2}}\left(\overrightarrow{A_{1}}-\overrightarrow{A_{2}}\right) \cdot\left(\nabla \bar{\phi}_{2}-\nabla \phi_{1}\right)\left(\omega_{1}+\bar{\omega}_{2}+\omega_{1} \bar{\omega}_{2}\right) d x \\
+\int_{\Omega} e^{i k x+\phi_{1}+\bar{\phi}_{2}}\left(\vec{A}_{1}^{2}-\vec{A}_{2}^{2}+q_{1}-q_{2}\right)\left(\omega_{1}+\bar{\omega}_{2}+\omega_{1} \bar{\omega}_{2}\right) d x
\end{gathered}
$$

If we fix $k, \gamma_{1}$, and $\gamma_{2}$, view $F_{1}$ and $F_{2}$ as functions of $s$ and apply (2.19) and (2.20) to (3.20) and (3.21), then it is clear that

$$
F_{1}=O(s), \quad F_{2}=O(1), \quad \text { as } s \rightarrow \infty .
$$

Thus

$$
\lim _{s \rightarrow \infty} s^{-1} F_{1}=-2 i \lim _{s \rightarrow \infty} s^{-1} \int_{\Omega} e^{i k x+\phi_{1}+\bar{\phi}_{2}} \xi \cdot\left(\overrightarrow{A_{1}}-\overrightarrow{A_{2}}\right) d x=0 .
$$

Since $\phi_{j}$ is continuous in $\xi_{j} /\left|\xi_{j}\right|$ (see (2.9)) and $\xi_{j} /\left|\xi_{j}\right|$ is continuous in $s$, it follows from (3.10), (3.11), (3.14), and (3.23) that

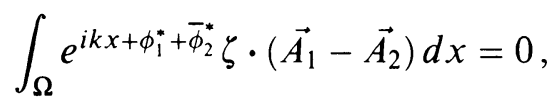

where $\phi_{j}^{*}=\phi_{j}^{*}(x, \zeta)$ solves

$$
\zeta \cdot \nabla \phi_{1}^{*}=-i \zeta \cdot \overrightarrow{A_{1}}, \quad \zeta \cdot \nabla \bar{\phi}_{2}^{*}=i \zeta \cdot \overrightarrow{A_{2}},
$$


and by (2.14)

$$
\begin{gathered}
\phi_{1}^{*}(x, \zeta)=\int_{\mathbb{R}^{2}} \frac{\zeta \cdot \overrightarrow{A_{1}}\left(x-\left(z_{1} \gamma_{1}+z_{2} \gamma_{2}\right)\right)}{z_{1}+i z_{2}} d z_{1} d z_{2}, \\
\bar{\phi}_{2}^{*}(x, \zeta)=-\int_{\mathbb{R}^{2}} \frac{\zeta \cdot \overrightarrow{A_{2}}\left(x-\left(z_{1} \gamma_{1}+z_{2} \gamma_{2}\right)\right)}{z_{1}+i z_{2}} d z_{1} d z_{2} .
\end{gathered}
$$

We summarize the result of this section in the following proposition.

Proposition 3.3. Let $\overrightarrow{A_{j}}$ and $q_{j}, j=1,2$, be potential functions satisfying (2.5). Assume that $\Lambda_{\overrightarrow{A_{1}}, q_{1}}=\Lambda_{\vec{A}_{2}, q_{2}}$. Then (3.24) holds with $\phi_{j}^{*}, j=1,2$, as in (3.26) and (3.27).

\section{PROOF OF THEOREM}

We divide this section into two parts. In the first part we shall prove $\operatorname{rot}\left(\overrightarrow{A_{1}}\right)=$ $\operatorname{rot}\left(\overrightarrow{A_{2}}\right)$ and in the second part we shall show $q_{1}=q_{2}$.

4.1. Proof of $\operatorname{rot}\left(\overrightarrow{A_{1}}\right)=\operatorname{rot}\left(\overrightarrow{A_{2}}\right)$. We shall assume throughout this subsection that $\Omega$ is a ball, i.e.,

$$
\Omega=\left\{x \in \mathbb{R}^{n},|x|<R\right\}
$$

for some $R>0$. This additional assumption would have no influence to our result. Suppose that $\Omega$ is not a ball, then we can choose a ball $\Omega^{\prime}$ so that $\Omega \subset \Omega^{\prime}$ and extend $\overrightarrow{A_{j}}$ and $q_{j}$ to be zero in $\Omega^{\prime} \backslash \Omega$. Clearly, $\overrightarrow{A_{j}}$ and $q_{j}$ still satisfy (2.5) with $\Omega=\Omega^{\prime}$ after the extension. Standard arguments show that $\Lambda_{\vec{A}_{1}, q_{1}}=\Lambda_{\overrightarrow{A_{2}}, q_{2}}$ on $\partial \Omega$ implies $\Lambda_{\vec{A}_{1}, q_{1}}=\Lambda_{\vec{A}_{2}, q_{2}}$ on $\partial \Omega^{\prime}$. Therefore it suffices to prove the theorem with assumption (4.1). See [SU-I] for relevant arguments.

Adding two equations in (3.25) together gives

$$
\zeta \cdot\left(\overrightarrow{A_{1}}-\overrightarrow{A_{2}}\right)=i \zeta \cdot \nabla\left(\phi_{1}^{*}+\bar{\phi}_{2}^{*}\right) .
$$

Substituting (4.2) into (3.24) and noticing $k \perp \zeta$ we have

$$
\begin{aligned}
\int_{\Omega} e^{i k x+\phi_{1}^{*}+\bar{\phi}_{2}^{*}} \zeta \cdot\left(\overrightarrow{A_{1}}-\overrightarrow{A_{2}}\right) d x & =i \int_{\Omega} e^{i k x} \zeta \cdot \nabla\left(e^{\phi_{1}^{*}+\bar{\phi}_{2}^{*}}\right) d x \\
& =i \int_{\Omega} \zeta \cdot \nabla\left(e^{i k x+\phi_{1}^{*}+\bar{\phi}_{2}^{*}}\right) d x .
\end{aligned}
$$

Then integrating by parts gives

$$
\int_{\partial \Omega} e^{i k x}(\zeta \cdot N) e^{\Psi(x, \zeta)} d s=0
$$

where

$$
\Psi(x, \zeta)=\phi_{1}^{*}(x, \zeta)+\bar{\phi}_{2}^{*}(x, \zeta)
$$

We shall use (4.3) to prove our result. We divide the remaining proof into three steps.

Step 1. For any $k \in \mathbb{R}^{n}, \zeta=\gamma_{1}+i \gamma_{2} \in \mathbb{C}^{n}$ with $\gamma_{1}$ and $\gamma_{2}$ as in (3.10) and integer $m \geq 0$,

$$
\int_{\partial \Omega} e^{i k x}(\zeta \cdot N)(\zeta \cdot x)^{m} e^{\Psi(x, \zeta)} d s=0
$$


Proof. Fix $\zeta=\gamma_{1}+i \gamma_{2}$ in (4.3). Since (4.3) holds for any $k \perp \gamma_{1}, \gamma_{2}$, it follows by using the inverse Fourier transform that

$$
\int_{\partial \Omega \cap T}(\zeta \cdot N) e^{\Psi(x, \zeta)} d s_{T}=0
$$

for any two-dimensional plane $T$ that is parallel to $\gamma_{1}$ and $\gamma_{2}$, where $d s_{T}$ is the usual surface measure on $\partial \Omega \cap T$.

Given $k, \gamma_{1}$, and $\gamma_{2}$, three mutually orthgonal vectors in $\mathbb{R}^{n}$ with $k \neq 0$ and $\left|\gamma_{1}\right|=\left|\gamma_{2}\right|=1$. We view $\left\{\gamma_{1}, \gamma_{2}, k\right\}$ as a right-handed three-dimensional frame in $\mathbb{R}^{n}$. Then

$$
\left\{\gamma_{1}, \gamma_{2}, k\right\} \rightarrow \int_{\partial \Omega} e^{i k x}(\zeta \cdot N) e^{\Psi(x, \zeta)} d s
$$

defines a map from the collection of all such frames to $\mathbb{C}$. We shall prove $(4.5)$ by differentiating this map in certain directions.

We construct two families of such frames, $\left\{\gamma_{1}^{(1)}(\theta), \gamma_{2}^{(1)}(\theta), k^{(1)}(\theta)\right\}$ and $\left\{\gamma_{1}^{(2)}(\theta), \gamma_{2}^{(2)}(\theta), k^{(2)}(\theta)\right\}$, depending smoothly on $\theta \in[0, \pi / 4]$, as follows. Define $\gamma_{2}^{(1)}(\theta)=\gamma_{2}$ for all $\theta$ and define $\gamma_{1}^{(1)}(\theta)$ and $k^{(1)}(\theta)$ to be the resulting position vectors after we rotate the (right-handed) two-dimensional frame $\left\{\gamma_{1}, k\right\}$ clockwise with an angle $\theta$ in the plane spanned by $\gamma_{1}$ and $k$. Similarly, define $\gamma_{1}^{(2)}(\theta)=\gamma_{1}$ for all $\theta$ and define $\gamma_{2}^{(2)}(\theta)$ and $k^{(2)}(\theta)$ to be the resulting position vectors after we rotate the (right-handed) two-dimensional frame $\left\{\gamma_{2}, k\right\}$ clockwise with an angle $\theta$ in the plane spanned by $\gamma_{2}$ and $k$. It is easy to show that

$$
\begin{gathered}
\left.\frac{d k^{(1)}}{d \theta}\right|_{\theta=0}=|k| \gamma_{1},\left.\quad \frac{d k^{(2)}}{d \theta}\right|_{\theta=0}=|k| \gamma_{2}, \\
\left.\frac{d \gamma_{1}^{(1)}}{d \theta}\right|_{\theta=0}=\left.\frac{d \gamma_{2}^{(2)}}{d \theta}\right|_{\theta=0}=-|k|^{-1} k,\left.\quad \frac{d \gamma_{2}^{(1)}}{d \theta}\right|_{\theta=0}=\left.\frac{d \gamma_{1}^{(2)}}{d \theta}\right|_{\theta=0}=0 .
\end{gathered}
$$

If we define

$$
\zeta^{(j)}(\theta)=\gamma_{1}^{(j)}(\theta)+i \gamma_{2}^{(j)}(\theta), \quad j=1,2,
$$

then (4.8) implies

$$
\left.\frac{d \zeta^{(1)}}{d \theta}\right|_{\theta=0}+\left.i \frac{d \zeta^{(2)}}{d \theta}\right|_{\theta=0}=0 .
$$

Next, we compute $\left.(d / d \theta)\left(\Psi\left(x, \zeta^{(1)}(\theta)\right)+i \Psi\left(x, \zeta^{(2)}(\theta)\right)\right)\right|_{\theta=0}$. Using formula (3.26) we have

$$
\Psi\left(x, \zeta^{(j)}(\theta)\right)=\int_{\mathbb{R}^{2}} \frac{\zeta^{(j)}(\theta) \cdot\left(\overrightarrow{A_{1}}-\overrightarrow{A_{2}}\right)\left(x-\left(z_{1} \gamma_{1}^{(j)}(\theta)+z_{2} \gamma_{2}^{(j)}(\theta)\right)\right)}{z_{1}+i z_{2}} d z_{1} d z_{2}
$$

for $j=1,2$. Using (4.8), (4.10), and (4.11) we obtain

$$
\begin{aligned}
\frac{d}{d \theta}( & \left.\Psi\left(x, \zeta^{(1)}(\theta)\right)+i \Psi\left(x, \zeta^{(2)}(\theta)\right)\right)\left.\right|_{\theta=0} \\
& =|k|^{-1} \int_{\mathbb{R}^{2}} k \cdot \nabla\left(\zeta \cdot\left(\overrightarrow{A_{1}}-\overrightarrow{A_{2}}\right)\right)\left(x-\left(z_{1} \gamma_{1}+z_{2} \gamma_{2}\right)\right) d z_{1} d z_{2} \\
& =|k|^{-1} k \cdot \nabla \int_{\mathbb{R}^{2}}\left(\zeta \cdot\left(\overrightarrow{A_{1}}-\overrightarrow{A_{2}}\right)\right)\left(x-\left(z_{1} \gamma_{1}+z_{2} \gamma_{2}\right)\right) d z_{1} d z_{2} .
\end{aligned}
$$


It is easy to see that the last integral in (4.12) is a function of $x$ which is equal to a constant on any two-dimensional plane that is parallel to $\gamma_{1}$ and $\gamma_{2}$.

Finally, letting $k=k^{(j)}(\theta)$ and $\zeta=\zeta^{(j)}(\theta), j=1,2$, in (4.3), differentiating with respect to $\theta$ and using (4.7) and (4.10), we get

$$
\begin{aligned}
0= & \left.\frac{d}{d \theta}\left(\int_{\partial \Omega} e^{i k^{(1)}(\theta) x}\left(\zeta^{(1)}(\theta) \cdot N\right) e^{\Psi\left(x, \zeta^{(1)}(\theta)\right)} d s\right)\right|_{\theta=0} \\
& +\left.i \frac{d}{d \theta}\left(\int_{\partial \Omega} e^{i k^{(2)}(\theta) x}\left(\zeta^{(2)}(\theta) \cdot N\right) e^{\Psi\left(x, \zeta^{(2)}(\theta)\right)} d s\right)\right|_{\theta=0} \\
= & |k| i \int_{\partial \Omega} e^{i k x}(\zeta \cdot N)(\zeta \cdot x) e^{\Psi(x, \zeta)} d s \\
& +\left.\int_{\partial \Omega} e^{i k x}(\zeta \cdot N) e^{\Psi(x, \zeta)} \frac{d}{d \theta}\left(\Psi\left(x, \zeta^{(1)}(\theta)\right)+i \Psi\left(x, \zeta^{(2)}(\theta)\right)\right)\right|_{\theta=0} d s .
\end{aligned}
$$

By (4.6) and (4.12)

$$
\begin{aligned}
\int_{\partial \Omega \cap T} & \left.(\zeta \cdot N) e^{\Psi(x, \zeta)} \frac{d}{d \theta}\left(\Psi\left(x, \zeta^{(1)}(\theta)\right)+i \Psi\left(x, \zeta^{(2)}(\theta)\right)\right)\right|_{\theta=0} d s_{T} \\
= & \left.\frac{d}{d \theta}\left(\Psi\left(x, \zeta^{(1)}(\theta)\right)+i \Psi\left(x, \zeta^{(2)}(\theta)\right)\right)\right|_{\theta=0} \int_{\partial \Omega \cap T}(\zeta \cdot N) e^{\Psi(x, \zeta)} d s_{T}=0
\end{aligned}
$$

for any two-dimensional plane $T$ that is parallel to $\gamma_{1}$ and $\gamma_{2}$. Therefore the last integral in (4.13) must be equal to zero. This proves (4.5) with $m=1$. Repeating the above procedure gives (4.5) with arbitrary positive integer $m$.

Step 2. Let $T$ be any two-dimensional plane that is parallel to $\gamma_{1}$ and $\gamma_{2}$. Then

$$
\int_{\partial \Omega \cap T}\left(\zeta \cdot N_{T}\right)^{m} \Psi(x, \zeta) d s_{T}=0
$$

for any integer $m \geq 1$, where $N_{T}$ is the outer normal of $\partial \Omega \cap T$ in $T$.

Proof. Using the same argument as the one which gave (4.6) we obtain from (4.5) that

$$
\int_{\partial \Omega \cap T}(\zeta \cdot N)(\zeta \cdot x)^{m} e^{\Psi(x, \zeta)} d s_{T}=0
$$

for any integer $m \geq 0$.

Recall that $\Omega=\left\{x \in \mathbb{R}^{n},|x|<R\right\}$ is a ball and thus $\partial \Omega \cap T$ is a circle with origin as its center in the plane $T$. Therefore

$$
\zeta \cdot N=\zeta \cdot N_{T}, \quad \zeta \cdot x=R\left(\zeta \cdot N_{T}\right)
$$

for $x \in \partial \Omega \cap T$. Combining (4.15) with (4.16) yields

$$
\int_{\partial \Omega \cap T}\left(\zeta \cdot N_{T}\right)^{m} e^{\Psi(x, \zeta)} d s_{T}=0
$$

for any integer $m \geq 1$.

If we denote by $\theta, 0 \leq \theta<2 \pi$, the angle between $\gamma_{1}$ and $N_{T}$, then $\zeta \cdot N_{T}=e^{i \theta}$. Hence (4.17) can be rewritten as

$$
\int_{0}^{2 \pi} e^{i m \theta} e^{f(\theta)} d \theta=0, \quad \forall \text { integer } m \geq 1,
$$


where $f=\left.\Psi\right|_{\partial \Omega \cap T}$. Equation (4.18) implies that there exists a holomorphic function $u$ defined on $D=\left\{x \in \mathbb{R}^{2} ;|x|<1\right\}$ such that $\left.u\right|_{\partial D}=e^{f}$. By the mapping property of the exponential function one sees that $\log u$ is well defined on $D$. Since $\log u$ is also holomorphic in $D$ and $\log u=f$ in $\partial D$, we must have

$$
\int_{0}^{2 \pi} e^{i m \theta} f(\theta) d \theta=0, \quad \forall \text { integer } m \geq 1,
$$

which leads to (4.17).

Step 3. $\operatorname{rot}\left(\overrightarrow{A_{1}}\right)=\operatorname{rot}\left(\overrightarrow{A_{2}}\right)$.

Proof. It suffices to show that

$$
\gamma_{1} \cdot \int_{\Omega} e^{i k x}\left(\overrightarrow{A_{1}}-\overrightarrow{A_{2}}\right) d x=0
$$

for $k$ and $\gamma_{1}$ as in (3.10). From (4.2) and (4.4) we have

$$
\gamma_{1} \cdot\left(\overrightarrow{A_{1}}-\overrightarrow{A_{2}}\right)=-\operatorname{Im}(\zeta \cdot \nabla \Psi) \text {. }
$$

Multiplying both sides of (4.21) by $e^{i k x}$ and integrating by parts we find that

$$
\gamma_{1} \cdot \int_{\Omega} e^{i k x}\left(\overrightarrow{A_{1}}-\overrightarrow{A_{2}}\right) d x=-\int_{\partial \Omega} e^{i k x} \operatorname{Im}((\zeta \cdot N) \Psi(x, \zeta)) d x .
$$

We now show that the right-hand side of (4.22) must be equal to zero. It suffices to show that

$$
\int_{\partial \Omega \cap T} \operatorname{Im}((\zeta \cdot N) \Psi(x, \zeta)) d s_{T}=0
$$

for any two-dimensional plane $T$ that is parallel to $\gamma_{1}$ and $\gamma_{2}$. Since this is just a consequence of (4.14) (with $m=1$ ), the proof is complete.

Corollary 4.1. There exists $p \in W_{\Omega}^{1, \infty}$ so that $\overrightarrow{A_{1}}-\overrightarrow{A_{2}}=\nabla p$ in $\Omega$.

Proof. $\operatorname{rot}\left(\overrightarrow{A_{1}}\right)=\operatorname{rot}\left(\overrightarrow{A_{2}}\right)$ implies that there exist $p \in W^{1, \infty}(\Omega)$ so that $\overrightarrow{A_{1}}-$ $\overrightarrow{A_{2}}=\nabla p$ in $\Omega$. The fact of $\operatorname{supp} \overrightarrow{A_{j}} \subset \bar{\Omega}, j=1,2$, implies that $p=$ constant in $\partial \Omega$. Hence, by substracting a constant we can adjust the function $p$ so that $p \in W_{\Omega}^{1, \infty}$.

4.2. Proof of $q_{1}=q_{2}$. From Corollary 4.1 and the fact that $\Lambda_{\vec{A}, q}$ is invariant under gauge transformations

$$
\vec{A} \rightarrow \vec{A}+\nabla p, \quad p \in W_{\Omega}^{1, \infty},
$$

we deduce that

$$
\Lambda_{\vec{A}_{1}, q_{2}}=\Lambda_{\vec{A}_{2}, q_{2}} .
$$

Then by the hypothesis we must have

$$
\Lambda_{\vec{A}_{1}, q_{1}}=\Lambda_{\vec{A}_{1}, q_{2}} \text {. }
$$

Thus, we may assume without loss of generality that $\overrightarrow{A_{1}}=\overrightarrow{A_{2}}=\vec{A} \in W_{\Omega}^{2, \infty}$ in the rest of this section. Under this assumption (3.9) reads

$$
\int_{\Omega}\left(q_{1}-q_{2}\right) u_{1} \bar{u}_{2} d x=0
$$


Substituting the solution (3.15) into (4.24) gives

(4.25) $\int_{\Omega} e^{i k x+\phi_{1}+\bar{\phi}_{2}}\left(q_{1}-q_{2}\right) d x=\int_{\Omega} e^{i k x+\phi_{1}+\bar{\phi}_{2}}\left(q_{1}-q_{2}\right)\left(\omega_{1}+\bar{\omega}_{2}+\omega_{1} \bar{\omega}_{2}\right) d x$.

From (4.2) and the statement following (3.23) we see that

$$
\phi_{1}+\bar{\phi}_{2} \rightarrow \phi_{1}^{*}+\bar{\phi}_{2}^{*}=0, \quad \text { in } L^{\infty}(\Omega) \quad \text { as } s \rightarrow \infty \text {. }
$$

Using this fact as well as (2.19) we deduce that the left-hand side of (2.25) tends to $\left(q_{1}-q_{2}\right)^{\wedge}(k)$ while the right-hand side tends to zero as $s$ goes to $\infty$. Therefore $q_{1}=q_{2}$ in $\Omega$.

\section{ACKNOWLEDGMENT}

It is a pleasure for the author to thank Victor Isakov for his interests in this work and for valuable suggestions. The author would also like to thank Adrian Nachman, John Sylvester, and Gunther Uhlmann for stimulating discussions.

\section{REFERENCES}

[A] G. Alessandrini, Stable determination of conductivity by boundary measurements, Appl. Anal. 27 (1988), 153-172.

[C] A. P. Calderon, On an inverse boundary value problem, Seminar on Numerical Analysis and Its Applications to Continuum Physics, Soc. Brasileira de Matematica, Rio de Janeiro, 1980, pp. 65-73.

[Ch] S. Chanillo, A problem in electrical prospection and an n-dimensional Borg-Levinson theorem, Proc. Amer. Math. Soc. 108 (1990), 761-767.

[I] V. Isakov, Completeness of products of solutions and some inverse problems for PDE, J. Differential Equations 92 (1991), 305-316.

[KV-I] R. Kohn and M. Vogelius, Determining conductivity by boundary measurements, Comm. Pure Appl. Math. 37 (1984), 289-298.

[KV-II] _ Determining conductivity by boundary measurements, Comm. Pure Appl. Math. 38 (1985), 643-667.

[LN] R. B. Lavine and A. Nachman, Global uniqueness in inverse problems with singular potentials, in preparation.

[N] A. Nachman, Reconstructions from boundary measurements, Ann. of Math. (2) 128 (1988), 531-576.

[NSU] A. Nachman, J. Sylvester, and G. Uhlmann, An n-dimensional Borg-Levinson theorem, Comm. Math. Phys. 115 (1988), 595-605.

[NH] R. Novikov and G. Henkin, $\bar{\partial}$-equation in the multidimensional inverse scattering problem, Uspekhi Mat. Nauk 42 (1987), no. 3, 93-152.

[R] A. G. Ramm, Recovery of the potential from fixed energy scattering data, Inverse Problems 4 (1988), 877-886.

[SU-I] J. Sylvester and G. Uhlmann, A global uniqueness theorem for an inverse boundary value problem, Ann. of Math. (2) 125 (1987), 153-169.

[SU-II] _ _ A uniqueness theorem for an inverse boundary value problem in electrical prospection, Comm. Pure Appl. Math. 39 (1986), 91-112.

[Su-I] Z. Sun, The inverse conductivity problems in two dimensions, J. Differential Equations 87 (1990), 227-255.

[Su-II] __, An inverse boundary value problem for the Schrödinger operator with vector potentials in two dimensions. Comm. Partial Differential Equations (to appear)

[SuU-I] Z. Sun and G. Uhlmann, Generic uniqueness for an inverse boundary value problem, Duke Math. J. 62 (1991), 131-155. 
[SuU-II] __, Inverse scattering for singular potentials in two dimensions, Trans. Amer. Math. Soc. 338 (1993), 363-374.

[W] Ricardo Weder, Global uniqueness at fixed energy in multidimensional inverse scattering theory, Inverse Problems 7 (1991), 927-938.

Department of Mathematics and Statistics, Wichita State University, Wichita, KANSAS 67208-1586

E-mail address: sun@twsuvm.bitnet 